\title{
Des plaquettes à la synovie, les métamorphoses d'un gène
}

Les membranes synoviales secrètent un liquide qui lubrifie les articulations et peut aussi éliminer certains métabolites qui s'accumulent dans les cavités articulaires. L'hyperplasie des synoviocytes, qui contribuent à la production de la synovie, s'observe dans l'arthrite rhumatoïde et dans les ostéoarthrites dont l'étiopathogénie est pourtant fort différente $(\mathrm{m} / \mathrm{s}$ 1995, $\left.n^{\circ} 11, p .1577\right)$. Elle s'observe aussi dans une maladie génétique récessive autosomique assez rare, associant des arthropathies non inflammatoires à une péricardite, appelée CACP (pour camptodactylie* - arthropathie - coxa vara** péricardite). Grâce à ce syndrome, dénommé aussi sérosite familiale fibrosante, on vient de découvrir un nouveau protéoglycane, sécrété dans la cavité articulaire, donc différent des protéoglycanes membranaires du cartilage (aggrécane, lumicane et fibromoduline, entre autres) [1]. Comme cela arrive de plus en plus souvent à présent que le génome a été largement exploré, il s'agit d'un gène déjà découvert dans d'autres circonstances. Tout a commencé en 1993, par l'identification, dans les urines de malades aplasiques en attente de greffe et présentant une thrombopénie importante, d'un facteur stimulant les mégacaryocytes (MSF). Le précurseur MSF est une protéine multifonctionnelle présente dans le cartilage articulaire et contenant des domaines homologues à la somatomédine $\mathrm{B}$, aux protéines liant l'héparine et aux mucines. L'année suivante, un groupe de Cardiff

\footnotetext{
* Contractures en flexion des articulations des doigts.

** Déformation angulaire de la hanche due à une anomalie de développement du col fémoral.
}

$\mathrm{m} / \mathrm{s} n^{\circ} 2$, vol. 16, février 2000
(Royaume-Uni) décrit un protéoglycane de $345 \mathrm{kDa}$, observé à la surface des cartilages articulaires de bovins [2]. Il est sécrété par les cellules synoviales et des chondrocytes de la zone superficielle du cartilage, d'où son nom: SZP (pour superficial zone protein). Tout récemment, afin d'élucider la structure complète de cette protéine, le même groupe constate que les ADNc de SZP de l'homme et des bovins présentent une homologie complète avec celui de MSF [3]. Les séquences entre bovins et humains sont identiques à $75 \%$, aussi bien en ce qui concerne les nucléotides que les acides aminés. Pendant ce tempslà, une équipe internationale s'efforçant de localiser le gène de la CACP à partir de familles, souvent consanguines, très éparpillées dans le monde (Arabie Saoudite, Canada, Terre Neuve, Mexique, Turquie) finit par conclure que le locus se trouvait en 1q25-q31 [4] et, par clonage positionnel et analyse des banques de données, il fut démontré que le gène, rebaptisé $C A C P$, n'était autre que le gène codant pour MSF et SZP [5]. Les mutations, observées chez huit malades atteints de CACP, sont des délétions, une transversion (qui crée un codon non-sens) et une insertion (entraînant une atteinte d'un site d'épissage). Elles doivent toutes avoir pour résultat la production d'une protéine tronquée. A l'état normal, cette protéine sécrétée pourrait avoir une action lubrificatrice, en raison de sa forte glycosylation et de la présence de répétitions de type mucine. Dans la CACP, l'hyperplasie serait alors la conséquence d'une insuffisance de lubrification des cellules de surface. Mais on peut aussi supposer que le gène $C A C P$ a une fonction régulatrice pri- maire des cellules de la couche intimale des articulations et du péricarde. A l'appui de cette hypothèse, la présence fréquente de coxa vara, de camptodactylies congénitales et de petits kystes remplis de matériel mucineux observés chez certains malades. La protéine CACP a peutêtre un rôle plus général au cours du développement car on ne connaît pas encore tous les tissus dans lesquels elle est présente. Enfin, il est possible que dans les arthrites rhumatoïdes, des mutations d'un tel gène puissent contribuer à l'aggravation des processus inflammatoires.

1. Praillet C, Grimaud J, Lortat-Jacob H. Les protéoglycanes: (II) rôles en pathologie. Med Sci $1998 ; 14: 421-8$.

2. Schumacher BL, Hughes CE, Kuettner KE, Caterson B, Aydelotte MB. Immunodetection and partial cDNA sequence of the proteoglycan, superficial zone protein, synthesized by cells lining synovial joints. J Orthop Res 1999; 17: 11020.

3. Flannery CR, Hughes CE, Schumacher BL, Tudor D, Aydelotte MB, et al. Articular cartilage superficial zone protein (SZP) is homologous to megakaryocyte stimulating factor precursor and is a multifunctional proteoglycan with potential growth-promoting, cytoprotective, and lubricating properties in cartilage metabolism. Biochem Biophys Res Commun 1999; 254: 535-41.

4. Bahabri SA, Suwairi WM, Laxer RM, Polinkovsky A, Dalaan AA, Warman ML. The camptodactyly-arthropathy-coxa vara-pericarditis syndrome: clinical features and genetic mapping to human chromosome 1. Arthritis Rheum 1998; 41 : 730-5.

5. Marcelino J, Carpten JD, Suwairi WM, Gutierrez OM, Schwartz S, et al. CACP, encoding a secreted proteoglycan, is mutated in camptodactylyarthropathy-coxa vara-pericarditis syndrome. Nat Genet 1999; 23: 319-22.

\section{Simone Gilgenkrantz \\ 9, rue Basse, 54330 Clerey-sur-Brenon, France.}

